\title{
Identification of Influential Variants in Significant Aggregate Rare Variant Tests
}

Rachel Z. Blumhagen ${ }^{1,2}$, David A. Schwartz ${ }^{3}$, Carl D. Langefeld ${ }^{4}$, Tasha E. Fingerlin ${ }^{1,2,3}$

${ }^{1}$ Center for Genes, Environment and Health, National Jewish Health, 1400 Jackson St., Denver, CO, 80206, USA

${ }^{2}$ Department of Biostatistics and Informatics, Colorado School of Public Health, 13001

E. 17th Place, Aurora, CO, 80045, USA

${ }^{3}$ School of Medicine, University of Colorado, 13001 E. 17th Place, Aurora, CO, 80045, USA

${ }^{4}$ Department of Biostatistics and Data Science, Wake Forest School of Medicine, Medical Center Boulevard, Winston-Salem, N.C., 27157, USA

Corresponding author: Rachel Blumhagen, National Jewish Health, 1400 Jackson St., Denver, CO, 80206, USA. email: rachel.blumhagen@cuanschutz.edu

Running title: Identification of Influential Rare Variants

Keywords: rare variants, localization, genetic association tests, idiopathic pulmonary fibrosis 


\section{Abstract}

3 Introduction: Studies that examine the role of rare variants in both simple and complex

4 disease are increasingly common. Though the usual approach of testing rare variants in

5 aggregate sets is more powerful than testing individual variants, it is of interest to

6 identify the variants that are plausible drivers of the association. We present a novel

7 method for prioritization of rare variants after a significant aggregate test by quantifying

8 the influence of the variant on the aggregate test of association.

10 Methods: In addition to providing a measure used to rank variants, we use outlier

11 detection methods to present the computationally efficient Rare Variant Influential

12 Filtering Iool (RIFT) to identify a subset of variants that influence the disease

13 association. We evaluated several outlier detection methods that vary based on the

14 underlying variance measure: interquartile range (Tukey fences), median absolute

15 deviation and standard deviation. We performed 1000 simulations for 50 regions of size

$163 \mathrm{~kb}$ and compared the true and false positive rates. We compared RIFT using the Inner

17 Tukey to two existing methods: adaptive combination of $p$-values (ADA) and a Bayesian

18 hierarchical model (BeviMed). Finally, we applied this method to data from our targeted

19 resequencing study in idiopathic pulmonary fibrosis (IPF).

21 Results: All outlier detection methods observed higher sensitivity to detect uncommon

22 variants $(0.001<\mathrm{MAF}>0.03)$ compared to very rare variants $(\mathrm{MAF}<0.001)$. For

23 uncommon variants, RIFT had a lower median false positive rate compared to the ADA. 
24 ADA and RIFT had significantly higher true positive rates than that observed for

25 BeviMed. When applied to two regions found previously associated with IPF including

26100 rare variants, we identified six polymorphisms with the greatest evidence for

27 influencing the association with IPF.

29 Discussion: In summary, RIFT has a high true positive rate while maintaining a low false

30 positive rate for identifying polymorphisms influencing rare variant association tests.

31 This work provides an approach to obtain greater resolution of the rare variant signals

32 within significant aggregate sets; this information can provide an objective measure to

33 prioritize variants for follow-up experimental studies and insight into the biological

34 pathways involved. 


\section{Introduction}

3 The number of studies that investigate the role of rare genetic variants in complex

4 diseases has been steadily increasing. This is in part due to the hypothesis that rare

5 variant effects might account for the discrepancy between estimated heritability for

6 complex traits and that accounted for by common variant associations [1]. In addition,

7 sequencing technologies have become less expensive such that more studies have the

8 ability to study rare variation in large numbers of individuals or families. A study of

9 circulating adiponectin levels found that among Hispanics, low frequency variants

10 explained more variation in the trait than common variants [2]. Rare variants have been

11 shown to have modest [3] or large effect sizes [1,4]. Rare variants can be associated

12 with increased [5] or decreased risk of disease [6]. Often rare variants influencing a trait

13 are only observed in a few families [7]. Thus, rare variants exhibit a range of association

14 patterns and can exhibit an important contribution to human trait variation.

16 Studies of rare variation often require either whole genome sequencing or targeted

17 sequencing on large study populations. Even with large sample sizes, there often

18 remains insufficient power for testing rare and uncommon variants individually $[8,9]$.

19 This is due to the low frequency of observations as well as the higher testing burden, as

20 there are far more rare variants compared to common variants in the genome. To

21 overcome this issue, methods for testing rare/uncommon variants often involve

22 aggregating information across a genomic region [10]. Variants may be grouped into

23 sets based on genomic information (e.g. gene bodies, exons), linkage disequilibrium 
1 blocks, association windows, or into overlapping sliding windows of a fixed size in terms

2 of physical distance or number of rare variants included. Methods for testing rare

3 variants in aggregate fall into three main types: burden tests, variance component tests,

4 and combined burden and variance component tests. The underlying assumption of

5 burden tests is that the effects of the rare variants within a given set are in the same

6 direction. Variance component tests ease this constraint and have higher power to

7 detect disease association with a mixture of protective and deleterious rare variants.

8 Given the underlying model by which rare variants are associated with disease in a

9 given region is unknown, tests which combine the burden and variance component

10 approaches weight the given contributions of the burden and variance components to

11 improve power.

13 Once a set of variants is found to be associated with a phenotype of interest, a logical

14 next step is the identification of the plausible drivers of the association that might be the

15 best statistical candidates for functional studies. Experimental validation of all rare

16 variants in a significant set is generally unreasonable based on time and expense. The

17 ability to narrow down the list of rare variants to those most likely to be contributing to

18 the association signal could help target experimental validation. Additionally, reporting

19 the rare variants most likely to be causal within a significant set of variants focuses

20 functional efforts and can aid in comparison of results across studies.

22 Several methods have been proposed for statistically identifying the most likely causal

23 variants [11-15]. One method uses a classic backward elimination procedure; a variant 
1 is removed from the set if its removal decreases the aggregate test $p$-value, and this

2 process is repeated until no improvement in the $p$-value from removing a variant is

3 observed [11]. Related stepwise procedures could be envisioned that use either the p-

4 value or various information criteria (e.g., Bayes information criteria, Akaike information

5 criterion). Another method considers the problem of prioritizing variants in aggregate

6 tests as a variable selection problem using kernel machine methods [16]. Although this

7 method was developed for prioritization of common variants rather than rare variants,

8 conceptually it is reasonable for rare variants. There are also previously published

9 methods which test each variant individually, such as using a Fisher's Exact Test and

10 applying the adaptive combination of $p$-values procedure (referred to as ADA) to the

11 resulting $p$-values [12]. This method has been shown to be more powerful than the

12 backward elimination method proposed by lonita-Laza, et. al., 2014. Most recently, the

13 BeviMed method applies a Bayesian hierarchical model and makes inference on

14 whether a rare variant is causal based on power posteriors [15]. Methods like the

15 backward elimination method and adaptive combination of $p$-values are iterative in

16 nature and therefore computationally intensive. In addition, the Bayesian approach is

17 developed for genome-wide testing of rare variant associations in rare Mendelian

18 disorders under specific (unknown) modes of inheritance rather than being more

19 broadly applicable.

21 We present a novel method for prioritization of rare variants within a given set of

22 variants after the set of variants is found to be significant using aggregate testing

23 methods. Building on the rich outlier-detection statistical literature, we present a 
1 computationally efficient approach to be applied following identification of a set of

2 variants that is agnostic to putative function. Our approach, which we refer to as RIFT

3 for Rare Variant Influential Filtering Iool, leverages the influence of the variant on the

4 aggregate test of association by quantifying the change in the aggregate test when that

5 variant is removed. It is particularly well suited for rare and uncommon variants, the

6 most common applications of aggregate tests, but is applicable to aggregate testing of

7 variants of all frequencies. When applied to a significant set of rare/uncommon variants,

8 RIFT provides a scheme for quantifying the contribution of an individual variant to the

9 overall association signal, while adjusting for covariates. This method also provides a

10 quantitative measure by which to rank variants for further investigation and several

11 visualizations to aid in evaluation of a region of interest.

\section{Methods}

15 Overview

16 We present the Rare Variant Influential Filtering Iool (RIFT) to quantify the effect of

17 each variant on an aggregate test of association (Figure 1). In our simulations, we

18 consider variants with a minor allele frequency (MAF) of $<3 \%$ (i.e., rare and

19 uncommon), but note that the method is directly applicable to any set of variants,

20 including common variants or mixtures of rare and common variants. First, we describe

21 our jackknife (leave-one-out) approach to obtain a score for each variant when applied

22 to a set of rare variants from an aggregate test. We then describe several outlier

23 detection methods that aim to identify influential variants (IVs) when applied to the 
1 variant scores within a set. We perform simulations to evaluate the jackknife scores and

2 the ability of the outlier detection methods to identify variants simulated to be associated

3 with the outcome. Finally, we apply RIFT to recently published rare variant regions

4 found to be significantly associated with idiopathic pulmonary fibrosis [17].

6 Localization Approach

7 For a given set of rare variants, we define the p-value resulting from the aggregate test

8 as $p_{(0)}$. For rare variant $j$, we calculate the $p$-value from the aggregate test of the

9 variants within the set excluding variant $j$ and refer to this $p$-value as $p_{(-j)}$. $P$-values are

10 transformed into chi-square statistics $(\mathrm{df}=1)$ using the inverse cumulative distribution

11 function (CDF) of the chi-square distribution (Equation 1), where $\chi_{(-j)}^{2}$ corresponds to

$12 p_{(-j)}$ and $\chi_{(0)}^{2}$ corresponds to $p_{(0)}$. For each rare variant $j$, we calculate a delta chi-

13 square score, denoted $\Delta \chi_{(-j)}^{2}$, which represents the change of the chi-square statistic

14 when the rare variant $j$ is removed (Equation 2). For variant $j, \Delta \chi_{(-j)}^{2}$ provides a relative

15 measure of how the results of the aggregate test compare with and without variant $j$.

16 Larger negative values indicate larger contributions to the overall test statistic.

18 Equation 1: Inverse CDF function of the chi-square distribution $(\mathrm{df}=1)$ can be written in

19 terms of the inverse CDF of the normal distribution, $N(0,1)$ denoted as $\Phi^{-1}$.

$$
\chi_{(-j)}^{2}=\left[\Phi^{-1}\left(\frac{p_{(-j)}+1}{2}\right)\right]^{2}
$$

21 Equation 2: Delta chi-square score

$$
\Delta \chi_{(-j)}^{2}=\chi_{(-j)}^{2}-\chi_{(0)}^{2}
$$


2 Outlier Detection for Identifying IVs

3 The delta chi-square score provides a quantitative measure to rank the rare variants

4 within a significant set according to the impact of that variant on the aggregate test of

5 association. In addition to ranking, it is also desirable to identify the subset of

6 polymorphisms influencing the set's statistical association. We considered this as an

7 outlier detection objective, whereby unusually large delta chi-square scores across a

8 significant set of variants correspond to the set of association influencing

9 polymorphisms. Sample values more extreme than a pre-specified cutoff are

10 determined to be outliers. Outlier detection methods are rooted in using an estimate of

11 the sample variance that is robust to outliers, and robust measures of spread are often

12 applied for that purpose. We considered two non-parametric approaches to identifying

13 outliers and compared these to a parametric approach.

15 Non-parametric variance estimation approaches

16 In 1977, Tukey defined the commonly-known descriptive univariate boxplot that displays

17 the interquartile range (IQR) as a measure of spread (IQR = distance between the first

18 [Q1] and third [Q3] quartiles; [18]. Boundaries based on the IQR are referred to as

19 "fences", and observations lying outside the fences are considered outliers. The inner

20 fence is defined by $Q 1-1.5^{\star} I Q R$ and $Q 3+1.5^{\star} I Q R$; the outer fence is defined by $Q 1$ -

$213^{*} \mid Q R$ and Q3 + 3*IQR. Note that the outer fence boundary is further away from the

22 median than that of the inner fence boundary, and is therefore more conservative in

23 classification of outliers. The IQR is robust to extreme values in the data and therefore 
1 identification of outliers using the IQR are superior to methods that rely on parametric

2 variance estimators in the presence of outliers [19]. Additionally, the IQR and

3 corresponding Tukey fences do not make any distributional assumptions and have been

4 shown to be effective as long as the data are not highly skewed [19].

6 Another measure of spread that does not assume a parametric distribution for the data

7 is the median absolute deviation (MAD). Identification of outliers based on the MAD is

8 provided in Equation 3 (further details can be found in Leys, Ley, Klein, Bernard, \&

9 Licata, 2013). Similar to the IQR, the MAD as a measure of spread is robust to extreme

10 values compared to the standard deviation, which is greatly influenced by extreme

11 values. The MAD requires specification of a constant and a cutoff; as others have used

12 for criteria in outlier detection, we set the constant corresponding to the normal

13 approximation $(b=1.4826)$ and a cutoff value of three; observations more than three

14 MAD away from the median are considered outliers [21,22].

15 Equation 3:

$$
M A D=b \cdot \operatorname{median}\left(\left|X_{j}-\tilde{X}\right|\right) ; \tilde{X}=\operatorname{median}(X)
$$

18 For completeness, we compare the cutoffs based on both the Inner and Outer Tukey

19 fences and MAD to that of the standard deviation (SD), in which observations more

20 extreme than three standard deviations from the mean are considered outliers. This

21 method is often referred to as the three sigma rule [23]. Given that we expect causal

22 variants to reduce the chi-square statistic when removed, we further limited

23 classification of IVs to variants having a negative delta chi-square score. 


\section{Simulated Data}

3 We follow a previously developed simulation approach to generate rare variant data

4 with a binary outcome $[24,25]$. Specifically, to generate case-control rare variant data

5 under both null and alternative hypotheses, we simulated 10,000 haplotypes for a $1 \mathrm{MB}$

6 genomic region under a coalescent model with parameters consistent with a European

7 population using the software package COSI [26]. We considered 50 different genomic

8 regions of size $3 \mathrm{~kb}$ and included only rare variants with $\mathrm{MAF}<0.03$. To generate

9 samples of cases and controls from the population, we repeatedly selected two

10 haplotypes at random and converted these haplotype data to genotypes at each variant

11 location. We determined the probability of subject $k$ being a case, defined as $p_{k}$, based

12 on a logistic regression equation where $\beta_{k}$ represents the coefficient for variant $k$

13 (Equation 4). $G_{j k}$ corresponds to number of minor alleles carried by subject $k$ for variant $j$

14 and $\beta_{0}$ the disease prevalence. For all simulations, the disease prevalence was fixed at

15 0.05. Case status, $Y_{k}$, is specified using the Bernoulli $\left(p_{k}\right)$ distribution.

16 Equation 4:

$$
\operatorname{logit}\left(p_{k}\right)=\beta_{0}+\sum_{j=1}^{J} \beta_{j} G_{j k}
$$

$$
Y_{k} \sim \operatorname{Bernoulli}\left(p_{k}\right)
$$

19 Consistent with previously published simulation of rare variant data, we defined the

20 coefficient for variants under the alternative (Equation 5) to be a function of the

21 population minor allele frequency $\left(M A F_{j}\right)$ for variant $j[24,25]$. This relationship between

22 the coefficient and the population MAF results in larger odds ratios for more rare 
1 variants. The constant, $c$, defines the strength of association between the causal

2 variants and the outcome.

3 Equation 5:

$$
\beta_{j}=c\left|\log _{10} M A F_{j}\right|
$$

5 With $c$ set to 0.4 , this corresponds to an odds ratio of 3.32 for a variant with a MAF of

$6 \quad 0.001$ and 7.39 for a variant with a MAF of 0.00001 . After determining case status for a

7 large sample of individuals, we sub-sampled to obtain a specified number of cases and

8 controls. As expected, the sub-sampling of the population results in some variants (both

9 under the null and alternative) to be no longer observed in a given sample.

11 Note that our approach can be applied to any such aggregate test (for a review of

12 methods for rare variant aggregate tests, please see Seunggeung Lee, Abecasis,

13 Boehnke, \& Lin, 2014). For exposition of the method, we use a combined burden and

14 variance component test, the Sequence Kernel Association Optimal Unified Test

15 (SKAT-O), due to its popularity in rare variant analyses [25]. We used the

16 recommended settings - including a linear weighted kernel, estimation of p-values using

17 the "davies" method and the variant weights as a function of the MAF using the

18 Beta $(1,25)$ distribution. If the SKAT-O p-value met the significance level (here, alpha

19 level of 0.05 ), we then applied our localization method to obtain a chi-square statistic

20 and delta chi-square score for each variant. We considered the proportion of the total

211000 samples with a SKAT-O p-value that met the significance as an estimate of the

22 power of SKAT-O for that region. SKAT-O performs a grid search to determine the

23 optimal value of the parameter $\rho$ that weights the relative influence of the burden and 
1 variance statistic. The parameter $\rho$ determined to be optimal for the full data was then

2 fixed when calculating the leave-one-out $p$-values. This insured that within a significant

3 set, the jackknife p-values and corresponding chi-square statistics are comparable. To

4 identify IVs, we applied the previously described outlier detection methods to the

5 resulting delta chi-square scores. For every variant, we calculated the proportion of

6 times it was labelled as an IV across the samples it was observed; for variants under

7 the alternative, this corresponds to a true positive rate and for variants under the null,

8 this corresponds to a false positive rate.

10 Comparison with existing methods

11 We compared the performance of RIFT to that of ADA and BeviMed by applying both

12 methods to simulated datasets. Due to the superior performance and computational

13 efficiency of ADA compared to the backward selection procedure, we chose to limit our

14 comparisons to these two methods [12]. We followed our simulation approach above,

15 where we simulated 50 genomic regions of size $3 \mathrm{~kb}$ with $10 \%$ of rare variants under the

16 alternative and effect size parameter of 0.4 . Given RIFT is developed for regions which

17 are previously found to be associated via an aggregate test of association, we restricted

18 our comparisons of methods to regions where the SKAT-O was significant at 0.05 . We

19 performed ADA using the default parameters: 1) a MAF threshold of $0.05,2$ ) calculation

20 of p-values using Fisher's exact test, 3) assuming an additive model and 4) 1000

21 permutations for calculation of the final region ADA test p-value. For each replicate, the

22 final set of variants (per-site p-value smaller than the optimal value) were classified as

23 IVs. We applied BeviMed using the default parameters: 1) for each variant, a prior 
1 probability of association of 0.01 and b) prior probability of a dominant model of

2 inheritance of 0.5 . BeviMed returns the posterior probability of association for both the

3 dominant and recessive models of inheritance. For each of these models, we classified

4 any variant with a posterior probability greater than 0.9 as a IV. We compared the above

5 methods to our localization method (RIFT) using both the Inner Tukey and MAD criteria

6 applied to the delta chi-square scores (referred to as RIFT:Inner Tukey and RIFT:MAD).

7 To compare the performance of the above methods, we calculated the true positive rate

8 and false positive rate across samples in which a variant was observed.

10 Impact of Varying Sample Characteristics on RIFT Performance

11 To determine the robustness of RIFT performance to varying characteristics of a given

12 sample, we performed additional simulations that varied the following parameters: \#

13 haplotypes simulated ( 1,000 and 100,000$)$, sample size (5,000 cases and 5,000

14 controls), region size $(0.75 \mathrm{~kb})$, disease prevalence $(10 \%)$, coefficient of disease

15 association $(c=0.8)$ and proportion of alternative variants $(20 \%)$. For each, we

16 simulated 500 samples for each of 10 regions while fixing the other simulation

17 parameters to those used in the comparisons with the ADA and BeviMed methods. We

18 summarized these different conditions by the structure of the resulting genotype data

19 (number of variants, etc.), number of samples meeting SKAT-O significance level

20 (power of SKAT-O) and finally in the performance of RIFT such as the relationship

21 between delta chi-square score, MAF and true positive rate (Supplemental Table 1).

22

23 Application to Rare Variant Regions Associated with IPF 
1 We applied our leave-one-out localization method to data from a recently published

2 targeted resequencing study in idiopathic pulmonary fibrosis (IPF) [27] as part of the

3 Global IPF Collaborative Network

4 (http://www.ucdenver.edu/academics/colleges/medicalschool/departments/medicine/Glo

5 ballPF/Pages/GloballPF.aspx). We applied RIFT with the goal of identifying rare

6 variants within regions associated with IPF to focus follow-up experimental validation

7 studies. We report results for two regions, each containing 50 rare variants. To provide

8 insight into putative function, we include functional annotation information obtained from

9 SNPDOC (https://wakegen.phs.wakehealth.edu/public/snpdoc3/index.cfm) and

10 HaploReg v4.1 [28].

12 Results

14 Characteristics of simulated regions

15 Among the 50 simulated $3 \mathrm{~kb}$ regions, each region included between 43 and 73 rare

16 variants (MAF < 3\%) with a median of 58.5 variants. Though we explored a range of the

17 proportion of variants assumed to be under the alternative, we report the results for

18 simulations where $10 \%$ of the variants in a given region were simulated to be under the

19 alternative. Results are qualitatively very similar for higher proportions of variants under

20 the alternative (Supplemental Table 2). After drawing random samples and selecting

211000 cases and 1000 controls to reflect the sampling process, the average proportion of

22 variants under the alternative across the 50 regions ranged from $9.7 \%$ to $15.2 \%$ with a

23 median of $12.3 \%$. Samples for a given region often contained higher than $10 \%$ 
1 alternative variants due to the over-sampling of cases to obtain an equal number of

2 cases and controls (Supplemental Table 2). We applied our localization method to

3 samples that had a SKAT-O p-value less than 0.05 . With the low proportion of variants

$4(10 \%)$ simulated under the alternative and effect size parameter, $c$, of 0.4 , the observed

5 median power of SKAT-O across the 1000 simulated samples was $22.0 \%$. As

6 expected, increasing the effect size parameter to 0.8 for the same 50 regions

7 dramatically increased the median power of SKAT-O to 99.1\% (Supplemental Table 2).

9 Performance of Our Localization Methods

10 Across all regions, we found an interesting relationship between the delta chi-square

11 score and the MAF (Figure 2). Delta chi-square scores were more extreme (and more

12 variable) for variants with smaller odds ratios and correspondingly higher MAFs. The

13 delta chi-square score is most sensitive to uncommon variants $(0.03>$ MAF $\geq 0.001)$

14 with modest effect sizes and least sensitive to rare variants (MAF < 0.001) with stronger

15 effect sizes. Positive delta chi-square scores were observed for some variants under the

16 alternative; however, the average delta chi-square score was negative for all but three

17 variants out of a total of 288 variants simulated under the alternative across the 50

18 regions. The general directionality of the delta chi-square score is consistent with what

19 we expect for causal variants, where the removal of a causal variant results in a larger

20 p-value, smaller chi-square statistic and therefore a negative delta chi-square score.

21 The relationship between the delta chi-square score and MAF was consistent when

22 varying the number of haplotypes simulated; however, we observed an even stronger 
1 relationship when increasing the sample size from 1,000 cases and controls to 5,000

2 cases and controls (Supplemental Figure 2).

4 Consistent with what was observed for the delta chi-square score, all outlier detection

5 methods observed higher sensitivity to detect uncommon variants $(0.03>M A F \geq 0.001)$

6 compared to very rare variants $(\mathrm{MAF}<0.001)$. The Inner Tukey fence had the highest

7 true positive rate (correctly labeling a variant under the alternative as an IV) compared

8 to other methods (Figure 3 ). For variants with a MAF $\geq 0.001$, corresponding to an odds

9 ratio less than 3.32, the Inner Tukey observed a median true positive rate of 0.60 (IQR:

$100.51,0.87$ ) compared to the SD method having a median of 0.30 (IQR: $0.10,0.59$;

11 Supplemental Table 4). Among the 288 variants under the alternative, the Inner Tukey

12 fence obtained the highest true positive rate $94.1 \%$ of the time and obtained a higher

13 true positive rate than the other three methods $74.7 \%$ of the time. The false positive rate

14 (inaccurately labeling a null variant as an IV) was remarkably low across all four

15 methods, with the SD method having the lowest rate. For variants with MAF $\geq 0.001$, the

16 SD method had a median false positive rate of 0 (IQR: 0,0$)$ and the Inner Tukey a

17 median of 0.09 (IQR: 0.06, 0.18; Supplemental Figure 1, Supplemental Table 4). Taken

18 together, the Inner Tukey has the best characteristics for correctly labeling a variant as

19 an IV, as evidenced by the high true positive rate and low false positive rate. The SD

20 method was substantially more conservative in labeling variants as IVs under the

21 alternative, especially for those variants having a MAF $\geq 0.0001$. 
Comparison with existing methods

2 Similar to RIFT, we observed a trend among existing localization methods in terms of

3 having increased ability to identify IVs at uncommon allele frequencies compared to

4 rare. (Figure 4, Table 1). While comparing the ADA and the BeviMed to the Inner Tukey

5 and MAD, the ADA had the highest true positive rate across the entire MAF spectrum

6 we considered, whereas BeviMed (dominant and recessive) observed the lowest true

7 positive rate. For uncommon variants ( $M A F \geq 0.001)$, the ADA had a median true

8 positive rate of 0.92 (IQR: $0.78,0.98)$ compared to BeviMed under the dominant model

9 having a median of 0.12 (IQR: $0.00,0.35)$. The higher sensitivity of the ADA to correctly

10 label IVs for rare MAFs is at the cost of having a high false positive rate (Figure 5;

11 median false positive rate of 0.22 [IQR: $0.17,0.34]$ ). As described above, the Inner

12 Tukey observed a lower median false positive rate of 0.09 (IQR: $0.06,0.18$ ). In

13 summary, both of our leave-one-out methods (Inner Tukey and MAD) produced a high

14 true positive rate for rare variants with MAF > 0.001 (comparable to ADA) while

15 maintaining low false positive rates across the entire spectrum of MAF.

17 Influential Variants in IPF Associated Loci

18 In a rare variant analysis of targeted sequencing in 3,017 idiopathic pulmonary fibrosis

19 (IPF) cases and 4,093 controls, we found several sets of rare variants associated with

20 IPF. Rare variants were grouped into gene-sets or sliding windows and tested for

21 association using SKAT-O. We applied RIFT to two significant rare variant windows,

22 each of which contained 50 rare variants. The most significant window is located on

23 chromosome 5 and spans the 5' UTR, exon 1 and intronic regions of the TERT gene. 
1 This window had a Bonferroni-adjusted SKAT-O p-value of $9.21 \times 10^{-16}$ after adjusting

2 for sex and the most strongly association common variant in the region, rs4449583.

3 After applying RIFT, there were three variants called outliers by both the Inner Tukey

4 fence and MAD cutoffs (Figure 6). Annotation with SNPDOC found the variant with the

5 largest delta chi-square (-28.7) to have unknown function and the second ranked IV

6 (delta chi-square $=-17.5$ ) to be in a non-coding RNA transcript in the 5 ' untranslated

7 region of TERT (Table 2). We additionally applied the localization method to a window

8 with a less significant association (SKAT-O Bonferroni-adjusted p-value = 0.0215;

9 adjusted for sex only as there is not a top common variant in the region) as an example

10 of a region with a more moderate aggregate rare variant association signal. This

11 window is located in the RTEL gene on chromosome 20 spanning both exons and

12 introns. Our localization method identified 3 IVs by both the Inner Tukey fence and MAD

13 cutoffs with the most outlying variant having a negative delta chi-square score $=-6.77$

14 (Figure 7; Table 2). This variant was annotated to be located in an intron of the RTEL

15 gene and based on annotation with HaploReg, has enhancer histone marks identified in

168 tissues including lung and a lung carcinoma cell line. The other two IVs were each

17 annotated to be in the coding region of RTEL and are nonsense mutations.

\section{Discussion}

21 The delta chi-square score we outline here provides an estimate of the contribution of a

22 given variant to the aggregate test statistic for a set of variants. This measure can be

23 used to rank variants in order to prioritize follow-up studies. We also compared several 
1 outlier detection methods to identify variants as having a disproportionate impact on the

2 aggregate test of association, likely increasing the probability of capturing a causal

3 variant. We found the inner Tukey fence to have the greatest sensitivity, and we

4 recommend this method to obtain a set of variants most likely to be driving the

5 aggregate signal. As expected, our method has higher sensitivity to detect uncommon

6 variants $(0.001<$ MAF $>0.03)$ compared to extremely rare variants (MAF < 0.001). This

7 underscores the difficulty in detecting extremely rare variants individually and thoughtful

8 alternative weighting schemes might provide leverage to better capture very rare

9 variants. The ADA method obtained the highest sensitivity for uncommon variants;

10 however, this was at a cost of low specificity. We found BeviMed lacked sufficient

11 sensitivity in the classification of IVs under our simulation framework. We recognize this

12 may be due to using their recommended parameter of 0.90 posterior probability for

13 classification of IVs. However, it is unclear how to determine an optimal value for the

14 posterior probability, which likely depends on the features of each region (e.g., number

15 of true causal variants, effect size of the variants, total number of variants tested).

16 Without requiring user optimization or selection of parameters, RIFT with the Inner

17 Tukey classification approach achieves higher sensitivity and specificity to ADA and

18 BeviMed, respectively.

20 The rare variants identified by our method to have the greatest evidence for influencing

21 the association with IPF have been previously found to be associated with other

22 diseases (Table 2). Common, non-coding variants in the promoter region of the TERT

23 gene have been found in several human cancers [29,30]. Specifically, the two IVs with 
1 the largest delta chi-square score (rs398123017 and rs373740199) have been identified

2 in studies of familial and sporadic melanoma and thyroid cancer [31-34]. These

3 mutations have been found to increase transcription of TERT and maintain telomerase

4 activity resulting in long telomeres, thereby promoting tumorigenesis [35].

6 On chromosome 20, two of the three rare variants identified by our method are

7 missense mutations in the RTEL gene and have been previously identified in

8 dyskeratosis congenita and the related Hoyeraal-Hreidarsson syndrome, both of which

9 are diseases that result due to failures in telomere maintenance [36-38]. Though

10 extensive research was not performed for all variants within our significant sets (as

11 we've previously noted is often prohibitive), identification of these variants in other

12 diseases supports the ability of our approach to detect plausible impactful rare variants

13 that drive our observed association with IPF.

15 Though we have only evaluated the delta chi-square statistic and corresponding outlier

16 detection methods for data containing unrelated individuals, RIFT is not restricted to

17 aggregate tests for this specific study design. Although we illustrated our approach

18 using SKAT-O in a case-control framework, the methods are widely applicable to other

19 aggregate tests and study designs. For example, the method could be applied using

20 tests developed for samples of related individuals such as famSKAT [39].

22 Unlike many other localization methods, RIFT does not rely on outside functional

23 information and is able to distinguish among variants with no known function that may 
1 be contributing to disease risk. We recognize that incorporating functional information

2 can increase the power to identify causal rare variants. However, methods which rely

3 on functional information are limited by the depth and accuracy of the annotation. Our

4 method complements methods that utilize functional information to provide increased

5 coverage in capturing plausible causal rare variation. Outlier detection methods to

6 identify influential variants will eventually break down if a large proportion of the variants

7 are under the alternative. It is plausible that in certain situations, the proportion of

8 variants that are under the alternative is higher for a set of variants that all have putative

9 function compared to a set that is selected agnostic of function. Since it is unlikely that

10 the majority of putatively functional variants in a gene are associated with a given trait, it

11 is also unlikely that filtering to functional variants prior to testing with an aggregate test

12 would be problematic for the outlier detection methods implemented in RIFT. While

13 RIFT maintains and perhaps even improves sensitivity for smaller numbers of variants

14 included in the aggregate test (Supplemental Table 5), pre-filtering variants after the

15 aggregate test is likely to be counter-productive. We recommend not filtering to variants

16 with putative function either before or after aggregate testing, but instead applying RIFT

17 agnostic of putative function and, if desired, further prioritizing those identified IVs based

18 on putative function.

$20 \mathrm{RIFT}$ is available as an R package from https://github.com/rachelzoeb/RIFT. The time to

21 complete an analysis with RIFT is dependent on the computation time of the aggregate

22 test used. SKAT-O is fast for sets with a relatively small number of variants (e.g., $<100$

23 rare variants) and requires parallelization for large significant sets. Future work will 
1 require optimization of RIFT for larger groups of variants such as sets based on gene

2 boundaries.

4 Greater resolution of rare variant signals within significant sets of variants can provide

5 valuable insight into the mechanism of disease and narrow the number of variants to

6 prioritize for follow-up experimental studies. Often, aggregate tests contain such a large

7 number of rare variants that follow up of each rare variant within the set would be

8 prohibitive, even after filtering down to variants with functional evidence for causality. In

9 addition, filtering to variants with known function precludes the ability to identify new

10 variants with as-yet unknown functional roles. In addition to being agnostic to functional

11 information, RIFT can be applied after application of any aggregate test, including those

12 that include common variants. The ease and flexibility of this approach will aid

13 investigators in post-aggregate association testing in a wide range of genetic studies. 


\section{ACKNOWLEDGEMENTS}

We acknowledge the Global IPF Collaborative Network (http://www.ucdenver.edu/academics/colleges/medicalschool/departments/medicine/Glo ballPF/Pages/GloballPF.aspx), the members of which contributed many of the DNA samples used to generate the resequencing data that are included in the example.

\section{STATEMENT OF ETHICS}

For the IPF resequencing data, all of the subjects provided written informed consent as part of institutional review board (IRB)- approved protocols for recruitment at their respective institution, and the resequencing study was approved by the National Jewish Health IRB and the University of Colorado Combined IRB.

\section{DISCLOSURE STATEMENT}

Dr. Schwartz reports personal fees from Eleven P15, Inc., outside the submitted work; In addition, Dr. Schwartz has a patent Compositions and Methods of Treating or Preventing Fibrotic Diseases pending, a patent Biomarkers for the diagnosis and treatment of fibrotic lung disease pending, and a patent Methods and Compositions for Risk Prediction, Diagnosis, Prognosis, and Treatment of Pulmonary Disorders issued. Dr. Fingerlin reports consulting fees from Eleven P15, Inc., outside the submitted work and a patent Methods and Compositions for Risk Prediction, Diagnosis, Prognosis, and Treatment of Pulmonary Disorders issued. All remaining authors besides Dr. Schwartz and Dr. Fingerlin declare no competing interest.

\section{FUNDING SOURCES}

This research was supported by the National Heart, Lung and Blood Institute (R01HL097163).

\section{AUTHOR'S CONTRIBUTIONS}

CDL, TEF, and RZB designed the study and developed the conceptual approaches to data analysis; TEF and DAS provided resequencing data. RZB performed the simulations and data analysis; RZB wrote the manuscript; TEF, DAS, and CDL reviewed the manuscript. 


\section{REFERENCES}

1. Bodmer W, Bonilla C. Common and rare variants in multifactorial susceptibility to common diseases. Nat Genet. 2008;40(6):695-701.

2. An SS, Palmer ND, Hanley AJG, Ziegler JT, Brown WM, Haffner SM, et al. Estimating the Contributions of Rare and Common Genetic Variations and Clinical Measures to a Model Trait: Adiponectin. Genet Epidemiol. 2013;37(1):13-24.

3. Sosnay PR, Siklosi KR, Van Goor F, Kaniecki K, Yu H, Sharma N, et al. Defining the disease liability of variants in the cystic fibrosis transmembrane conductance regulator gene. Nat Genet. 2013;45(10):1160-7.

4. Singh T, Walters JTR, Johnstone M, Curtis D, Suvisaari J, Torniainen M, et al. The contribution of rare variants to risk of schizophrenia in individuals with and without intellectual disability. Nat Genet. 2017;49(8):1167-73.

5. Fearnhead NS, Wilding JL, Winney B, Tonks S, Bartlett S, Bicknell DC, et al. Multiple rare variants in different genes account for multifactorial inherited susceptibility to colorectal adenomas. Proc Natl Acad Sci U S A. 2004;101(45):15992-7.

6. He KY, Li X, Kelly TN, Liang J, Cade BE, Assimes TL, et al. Leveraging linkage evidence to identify low-frequency and rare variants on $16 \mathrm{p} 13$ associated with blood pressure using TOPMed whole genome sequencing data. Hum Genet. 2019;0(0):0.

7. Armanios MY, Chen JJL, Cogan JD, Alder JK, Ingersoll RG, Markin C, et al. Telomerase mutations in families with idiopathic pulmonary fibrosis. $\mathrm{N}$ Engl $\mathrm{J}$ Med. 2007;356(13):1317-26.

8. Lee S, Abecasis GR, Boehnke M, Lin X. Rare-Variant Association Analysis: Study Designs and Statistical Tests. Am J Hum Genet. 2014 Jul;95(1):5-23.

9. Cruchaga C, Karch CM, Jin SC, Benitez BA, Cai Y, Guerreiro R, et al. Rare coding variants in the phospholipase D3 gene confer risk for Alzheimer's disease. Nature. 2014 Jan 11;505(7484):550-4.

10. Bansal V, Libiger O, Torkamani A, Schork NJ. Statistical analysis strategies for association studies involving rare variants. Nat Rev Genet. 2010;11(11):773-85.

11. Ionita-Laza I, Capanu M, De Rubeis S, McCallum K, Buxbaum JD. Identification of Rare Causal Variants in Sequence-Based Studies: Methods and Applications to VPS13B, a Gene Involved in Cohen Syndrome and Autism. PLoS Genet. 2014;10(12).

12. Lin WY. Beyond Rare-Variant Association Testing: Pinpointing Rare Causal Variants in Case-Control Sequencing Study. Sci Rep. 2016;6(September 2015):1-13.

13. He Q, Almli LM, Conneely KN, Zhao N, Ressler KJ, Binder EB, et al. Prioritizing individual genetic variants after kernel machine testing using variable selection. 2016;(August):722-31.

14. Sun H, Wang S. A power set-based statistical selection procedure to locate susceptible rare variants associated with complex traits with sequencing data. Bioinformatics. 2014;30(16):2317-23.

15. Greene D, Richardson S, Turro E. A Fast Association Test for Identifying 
Pathogenic Variants Involved in Rare Diseases. Am J Hum Genet. 2017;101(1):104-14.

16. He Q, Cai T, Liu Y, Zhao N, Harmon QE, Almli LM, et al. Prioritizing individual genetic variants after kernel machine testing using variable selection. Genet Epidemiol. 2016;40(8):722-31.

17. Moore C, Blumhagen RZ, Yang I V, Walts A, Powers J, Walker T, et al. Resequencing Study Confirms Host Defense and Cell Senescence Gene Variants Contribute to the Risk of Idiopathic Pulmonary Fibrosis. Am J Respir Crit Care Med. 2019 Apr 29;rccm.201810-18910C.

18. Tukey JW. Exploratory Data Analysis. Addison-Wesley Publishing Company; 1977. (Addison-Wesley series in behavioral science).

19. Seo S, Gary M. Marsh PD. A review and comparison of methods for detecting outliers in univariate data sets. Dep Biostat Grad Sch Public Heal. 2006;53+7.

20. Leys C, Ley C, Klein O, Bernard P, Licata L. Detecting outliers: Do not use standard deviation around the mean, use absolute deviation around the median. $\mathrm{J}$ Exp Soc Psychol. 2013;49(4):764-6.

21. Rousseeuw PJ, Croux C. Alternatives to the Median Absolute Deviation. J Am Stat Assoc. 1993;88(424):1273.

22. Jones PR. A note on detecting statistical outliers in psychophysical data. Attention, Perception, Psychophys. 2019;1189-96.

23. Pukelsheim F. The Three Sigma Rule. Am Stat. 1994 May;48(2):88.

24. Wu MC, Lee S, Cai T, Li Y, Boehnke M, Lin X. Rare-variant association testing for sequencing data with the sequence kernel association test. Am J Hum Genet. 2011;89(1):82-93.

25. Lee S, Emond MJ, Bamshad MJ, Barnes KC, Rieder MJ, Nickerson DA, et al. Optimal Unified Approach for Rare-Variant Association Testing with Application to Small-Sample Case-Control Whole-Exome Sequencing Studies. Am J Hum Genet. 2012;91(2):224-37.

26. Schaffner S, Foo C, Gabriel S. Calibrating a coalescent simulation of human genome sequence variation. Genome .... 2005;1576-83.

27. Moore C, Blumhagen RZ, Yang I V., Walts A, Schwartz DA, Fingerlin TE. Resequencing Study Confirms Host Defense and Cell Senescence Gene Variants Contribute to the Risk of Idiopathic Pulmonary Fibrosis. Am J Respir Crit Care Med. 2019;

28. Ward LD, Kellis M. HaploReg: A resource for exploring chromatin states, conservation, and regulatory motif alterations within sets of genetically linked variants. Nucleic Acids Res. 2012;40(D1):930-4.

29. Bojesen SE, Pooley KA, Johnatty SE, Beesley J, Michailidou K, Tyrer JP, et al. Multiple independent variants at the TERT locus are associated with telomere length and risks of breast and ovarian cancer. Nat Genet. 2013;45(4):371-84.

30. Haiman CA, Chen GK, Vachon CM, Canzian F, Dunning A, Millikan RC, et al. A common variant at the TERT-CLPTM1L locus is associated with estrogen receptor-negative breast cancer. Nat Genet. 2011 Dec 30;43(12):1210-4.

31. Horn S, Figl A, Rachakonda PS, Fischer C, Sucker A, Gast A, et al. TERT Promoter Mutations in Familial and Sporadic Melanoma. Science (80- ). 2013 Feb 22;339(6122):959-61. 
32. Huang FW, Hodis E, Xu MJ, Kryukov G V., Chin L, Garraway LA. Highly Recurrent TERT Promoter Mutations in Human Melanoma. Science (80- ). 2013 Feb 22;339(6122):957-9.

33. Harland M, Petljak M, Robles-Espinoza CD, Ding Z, Gruis NA, van Doorn R, et al. Germline TERT promoter mutations are rare in familial melanoma. Fam Cancer. 2016;15(1):139-44.

34. Liu R, Xing M. TERT promoter mutations in thyroid cancer. Endocr Relat Cancer. 2016 Mar;23(3):R143-55.

35. Chiba K, Johnson JZ, Vogan JM, Wagner T, Boyle JM, Hockemeyer D. Cancerassociated tert promoter mutations abrogate telomerase silencing. Elife. 2015;4(JULY 2015):1-20.

36. Ballew BJ, Yeager M, Jacobs K, Giri N, Boland J, Burdett L, et al. Germline mutations of regulator of telomere elongation helicase 1, RTEL1, in Dyskeratosis congenita. Hum Genet. 2013 Apr 18;132(4):473-80.

37. Walne AJ, Vulliamy T, Kirwan M, Plagnol V, Dokal I. Constitutional mutations in RTEL1 cause severe dyskeratosis congenita. Am J Hum Genet. 2013;92(3):44853.

38. Deng Z, Glousker G, Molczan A, Fox AJ, Lamm N, Dheekollu J, et al. Inherited mutations in the helicase RTEL1 cause telomere dysfunction and HoyeraalHreidarsson syndrome. Proc Natl Acad Sci. 2013;110(36):E3408-16.

39. Chen H, Meigs JB, Dupuis J. Sequence Kernel Association Test for Quantitative Traits in Family Samples. Genet Epidemiol. 2013 Feb;37(2):196-204. 


\section{TABLES}

Table 1. Summary of true positive and false positive rates, stratified by MAF for each localization method. The outlier detection methods for RIFT (MAD and Inner Tukey) were applied to the delta chi-square scores. Simulation included 1000 samples per region, across 50 regions. Data were simulated to have $10 \%$ variants under the alternative with effect size parameter $c=0.4$ (see Equation 5).

\begin{tabular}{ccccc}
\hline \multirow{2}{*}{ Method } & \multicolumn{2}{c}{ True Positive Rate - Median (IQR) } & \multicolumn{2}{c}{ False Positive Rate - Median (IQR) } \\
& MAF $<0.001$ & MAF $\geq 0.001$ & MAF $<0.001$ & MAF $\geq 0.001$ \\
\hline RIFT:MAD & $0.03(0.01,0.11)$ & $0.54(0.43,0.85)$ & $0.00(0.00,0.01)$ & $0.08(0.04,0.15)$ \\
\hline RIFT:Inner Tukey & $0.04(0.01,0.15)$ & $0.60(0.51,0.87)$ & $0.00(0.00,0.01)$ & $0.09(0.06,0.18)$ \\
\hline BeviMed:DOM & $0.00(0.00,0.00)$ & $0.12(0.00,0.35)$ & $0.00(0.00,0.00)$ & $0.00(0.00,0.00)$ \\
\hline BeviMed:REC & $0.00(0.00,0.00)$ & $0.02(0.00,0.27)$ & $0.00(0.00,0.00)$ & $0.00(0.00,0.01)$ \\
\hline ADA & $0.17(0.08,0.35)$ & $0.92(0.78,0.98)$ & $0.01(0.00,0.05)$ & $0.22(0.17,0.34)$ \\
\hline
\end{tabular}


Table 2. Influential Variants identified in two rare variant loci previously found associated with IPF

\begin{tabular}{|c|c|c|c|c|c|c|c|}
\hline Chr:Pos $^{a}$ & MA $^{\mathbf{b}}$ & MAF $^{c}$ & $\begin{array}{c}\text { Delta Chi- } \\
\text { Square }\end{array}$ & rs\# & SNP-DOC annotation & $\begin{array}{c}\text { Nearest } \\
\text { Gene }\end{array}$ & Publications/Clinical Notes \\
\hline chr5:1294704 & A & 0.00056 & -3.65 & & coding-synon,ncRNA & TERT & \\
\hline chr5:1295161 & G & 0.00353 & -17.50 & rs878855297 & ncRNA, untranslated-5 & TERT & $\begin{array}{ll}- & \text { Familial and sporadic melanoma (Horn et al., 2013) } \\
\text { - } & \text { High penetrance, early onset melanoma (Harland et } \\
\text { al., 2016) } \\
\text { - } \quad \text { Cancer cell lines, found to abrogate telomerase } \\
\text { silencing and promote tumorigenesis (Chiba et al., } \\
\text { 2015) }\end{array}$ \\
\hline chr5:1295228 & $\mathrm{A}$ & 0.00705 & -28.68 & & unknown & TERT & $\begin{array}{ll}\text { - } & \text { Familial and sporadic melanoma (Horn et al., 2013; } \\
\text { - } & \text { Huang et al., 2013) } \\
\text { Cancer cell lines, found to abrogate telomerase } \\
\text { silencing and promote tumorigenesis (Chiba et al., } \\
\text { 2015) } \\
\text { - } \quad \text { Thyroid cancer (Liu and Xing, 2016) }\end{array}$ \\
\hline chr20:62324391 & $\mathrm{A}$ & 0.05008 & -6.77 & rs41308092 & intron & RTEL & \\
\hline chr20:62324564 & $\mathrm{T}$ & 0.00197 & -3.25 & rs398123017 & ncRNA, nonsense & RTEL & $\begin{array}{ll}\text { - } & \text { Dyskeratosis congenital (Ballew et al., 2013; Walne } \\
& \text { et al., 2013) } \\
\text { - } & \text { Hoyeraal-Hreidarsson syndrome (Deng et al., 2013) } \\
\text { - } & \text { Familial interstitial pneumonia (Cogan et al., 2015) } \\
\text { - } & \text { Idiopathic pulmonary fibrosis (Todd et al., 2017) }\end{array}$ \\
\hline chr20:62324600 & $\mathrm{T}$ & 0.00098 & -2.60 & rs373740199 & coding-synon,ncRNA,nonsense & RTEL & $\begin{array}{ll}- & \text { Dyskeratosis congenital (Ballew et al., 2013) } \\
\text { - } & \text { Hoyeraal-Hreidarsson syndrome (Moriya et al., } \\
\text { 2016) } \\
\text { - } \quad \text { Idiopathic pulmonary fibrosis (Todd et al., 2017) } \\
\text { - } \quad \text { Early-onset Inflammatory Bowel Disease (Petersen } \\
\text { et al., 2017) }\end{array}$ \\
\hline
\end{tabular}

${ }^{a} \mathrm{NCBI}$ Build 37 coordinates

${ }^{\mathrm{b}}$ Minor allele observed in our data

cMinor allele frequency observed in our data

${ }^{\mathrm{d}}$ HaploReg results: promoter histone marks found in skin, $\mathrm{Gl}$ and enhancer histone marks found in 8 tissues including lung and a lung carcinoma cell line 


\section{FIGURES}

Figure 1. Flow chart of RIFT.

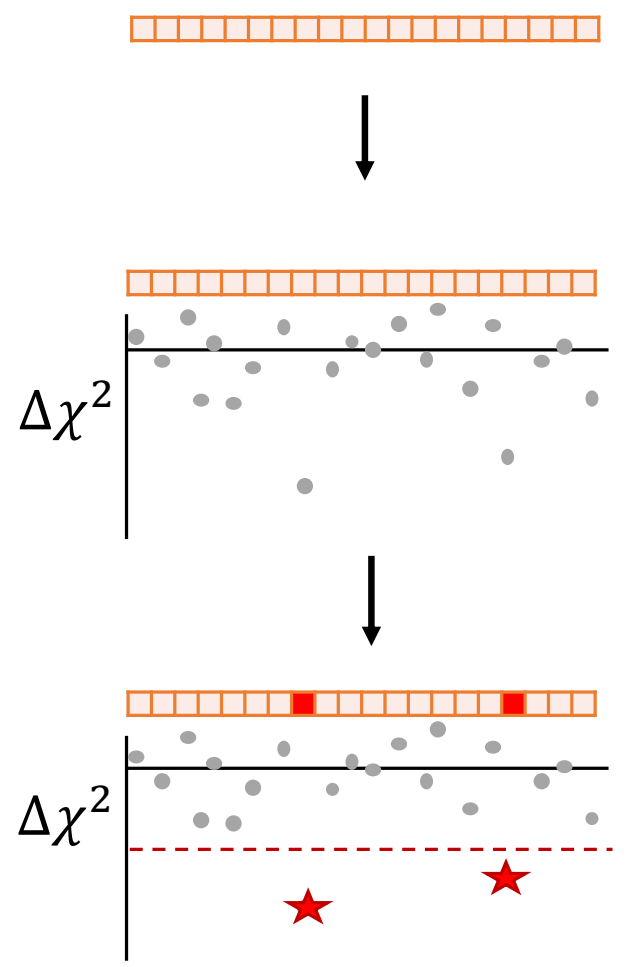

Identify set of rare variants via aggregate testing (e.g. SKAT-O)

\section{STEP 1}

For each variant, obtain delta chisquare scores via leave-one-out approach

\section{STEP 2}

To identify influential variants, apply outlier detection methods to set of delta chi-square scores

Methods: Tukey Fences, MAD or SD 
Figure 2. Delta chi-square score more sensitive to the more frequently observed rare variants with correspondingly smaller effect sizes. Mean delta chi-square score plotted by odds ratio for variants simulated under the alternative across all 50 regions. Data were simulated to have $10 \%$ variants under the alternative with effect size parameter $c=0.4$ (see Equation 5).

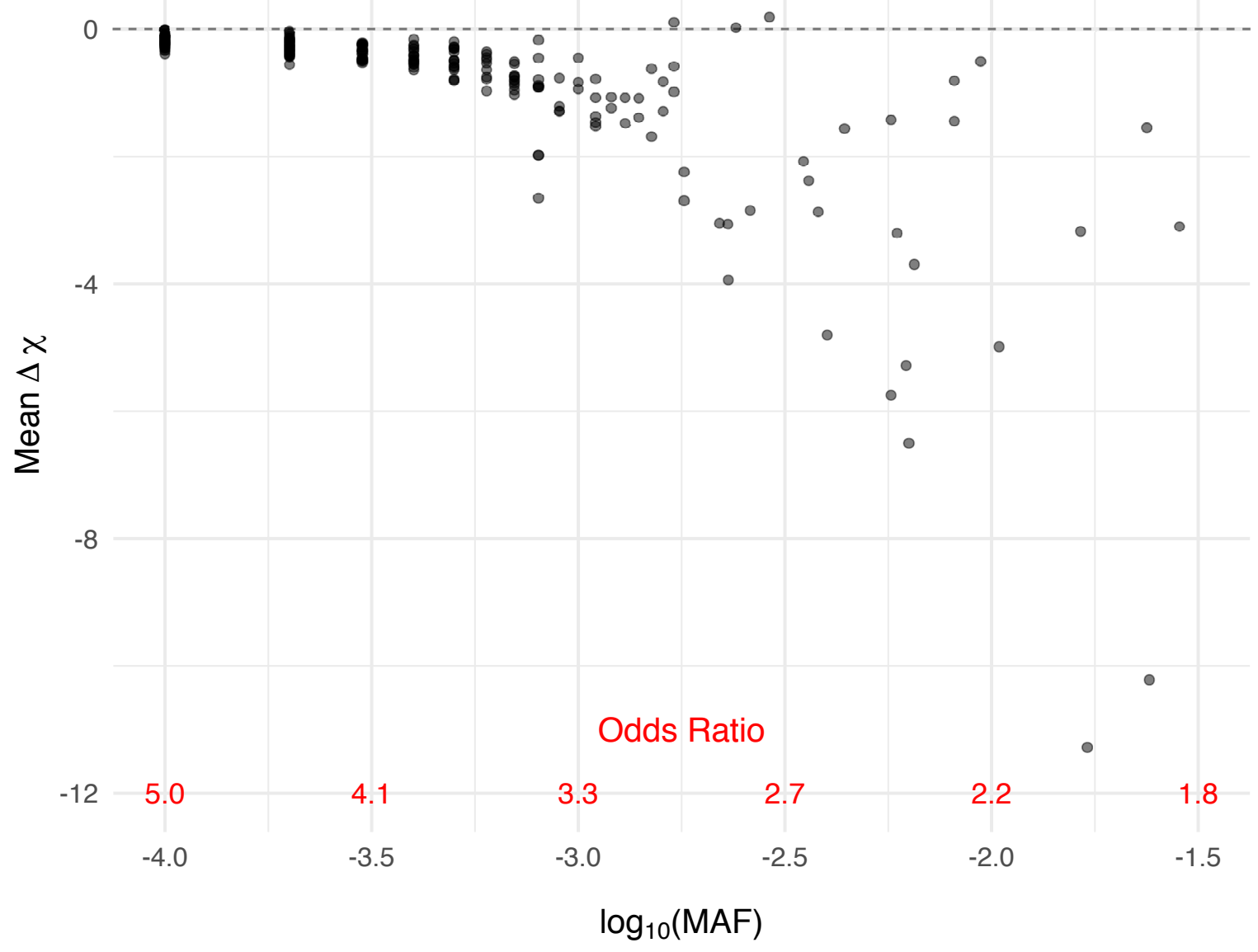


Figure 3. True positive rate (proportion correctly labeled IV) for variants under the alternative for each outlier detection method of RIFT as a function of MAF. Corresponding odds ratio is also provided for reference. Smoothed line and confidence band provided by the loess method. Data were simulated to have $10 \%$ variants under the alternative with effect size parameter $c=0.4$ (see Equation 5).

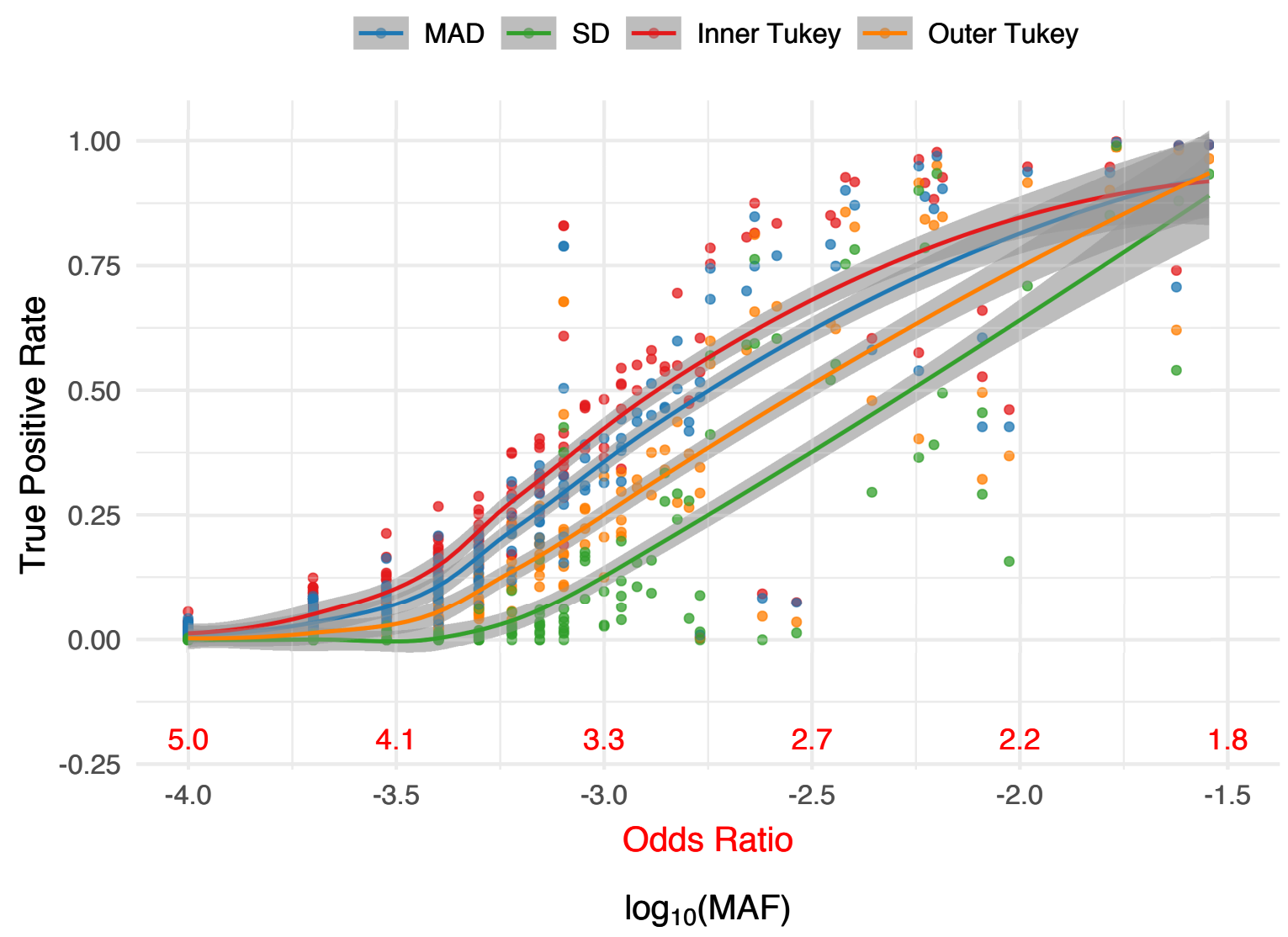


Figure 4. True positive rate (proportion correctly labeled IV) for variants under the alternative for each localization method as a function of MAF. Corresponding odds ratio is also provided for reference. Smoothed line and confidence band provided by the loess method. Data were simulated to have $10 \%$ variants under the alternative with effect size parameter $c=0.4$ (see Equation 5).

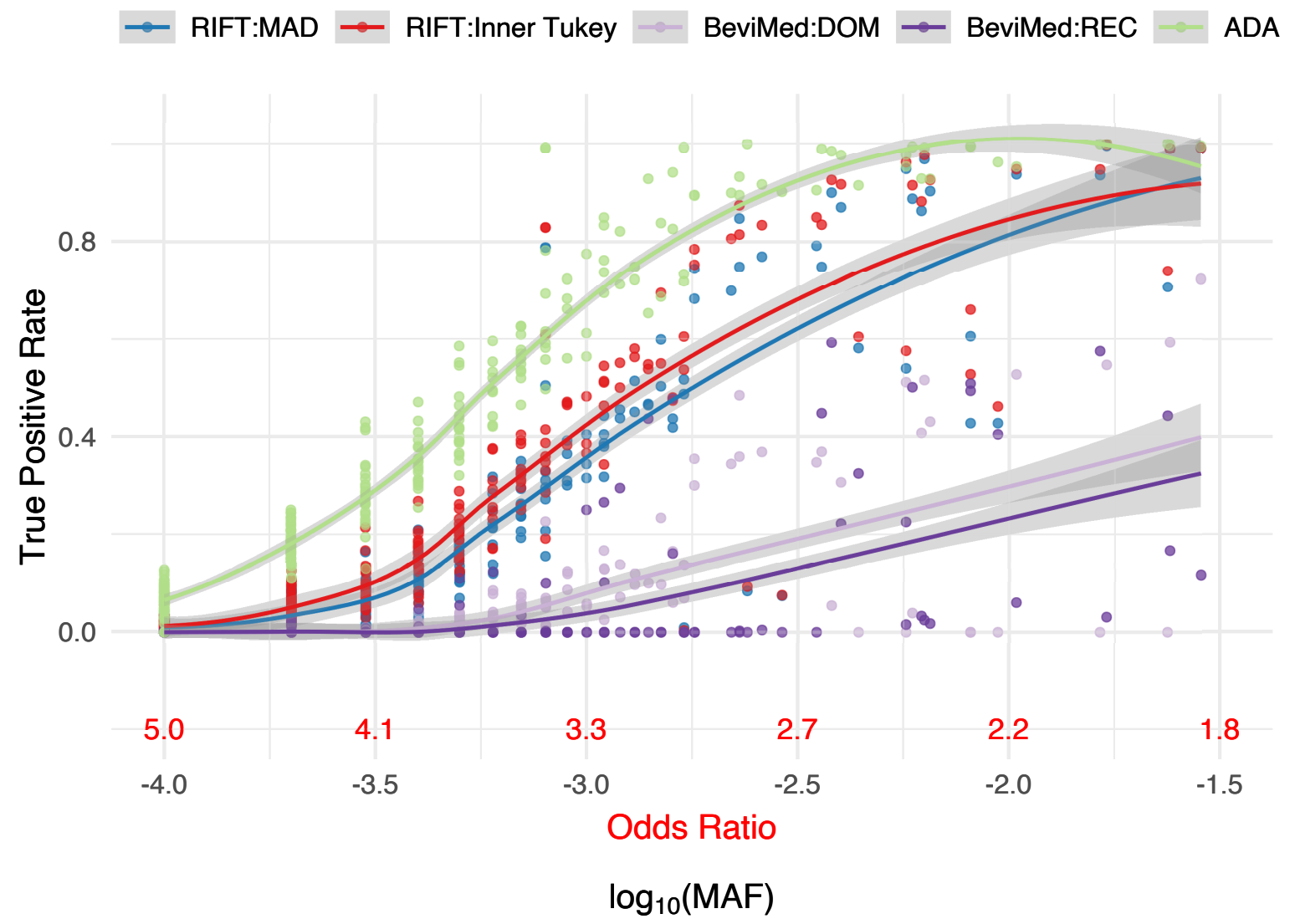


Figure 5. False positive rate (proportion incorrectly labeled IV) for variants under the null for each localization method as a function of MAF. Smoothed line and confidence band provided by the loess method. Data were simulated to have $10 \%$ variants under the alternative with effect size parameter $c=0.4$ (see Equation 5).

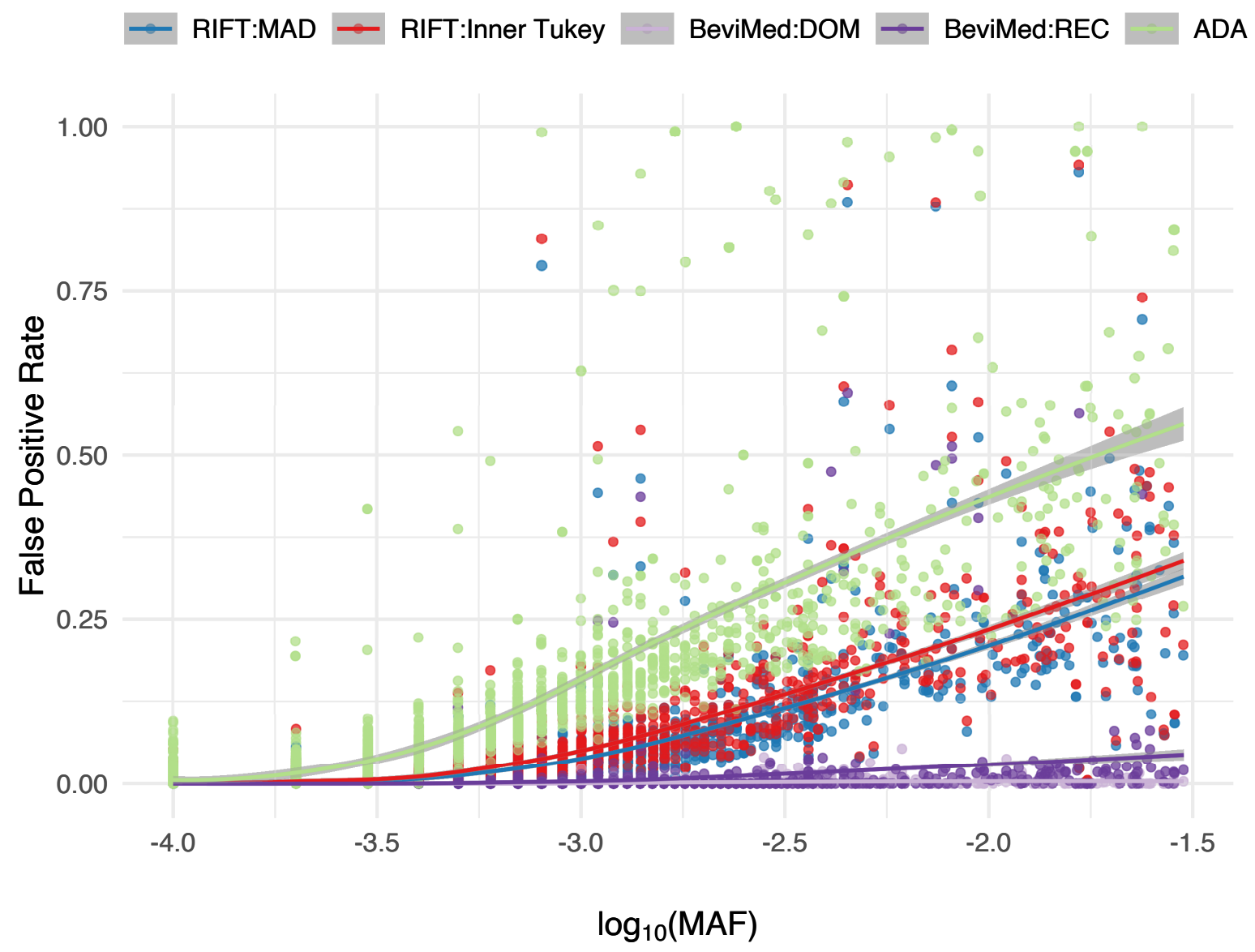


Figure 6. Chi-square (top and delta chi-square scores (bottom) by genomic position for the IPF-associated rare variant loci on chr5 (bp: 1294397-1295255). Color for the top plot corresponds to SNP-DOC functional annotation and for the bottom plot, color corresponds to outlier by the Inner Tukey method and shape corresponds to outlier by the MAD method.

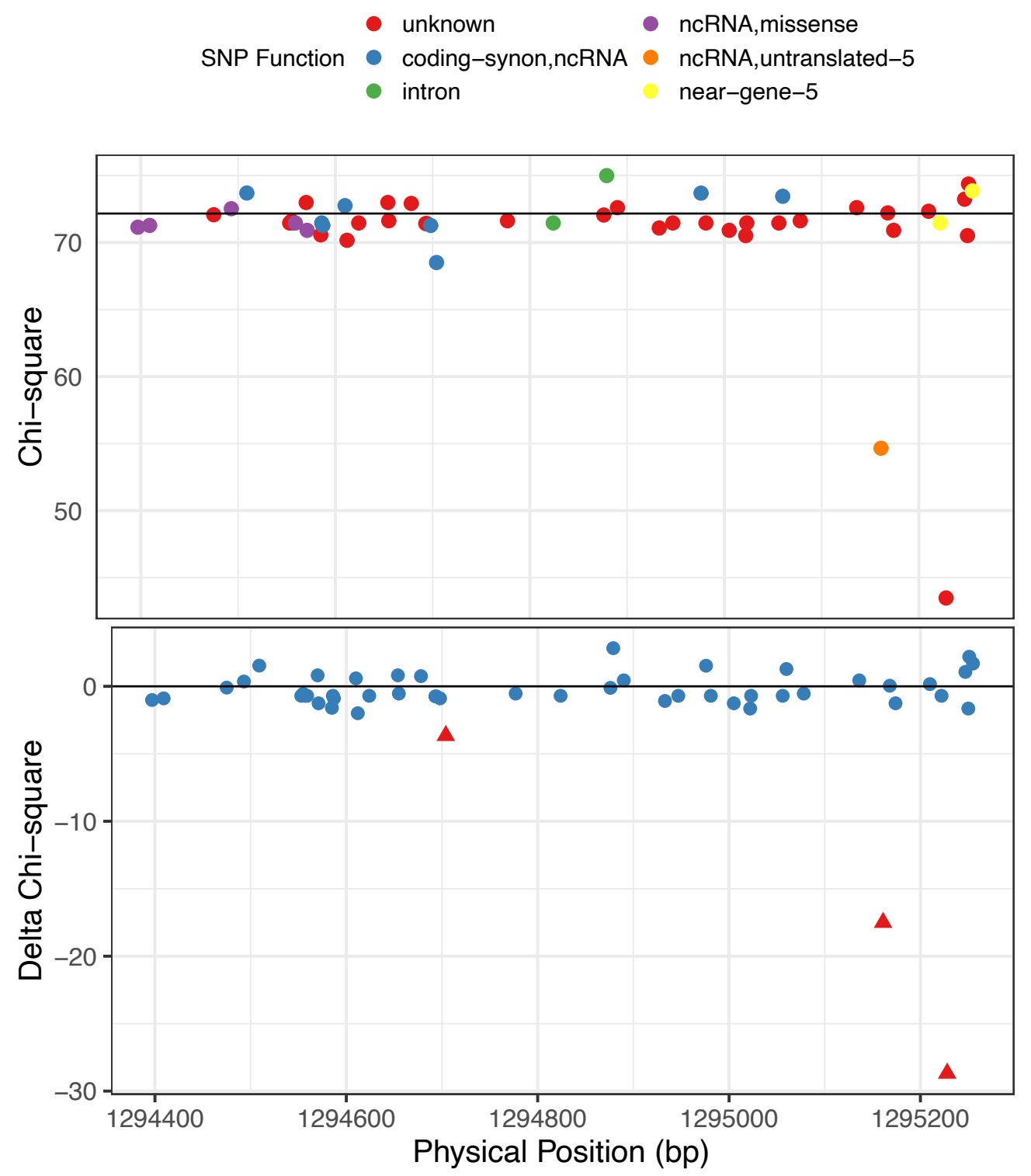

RIFT:Inner Tukey $\quad$ Not Outlier Outlier

RIFT:MAD • Not Outlier

- Outlier 
Figure 7. Chi-square (top and delta chi-square scores (bottom) by genomic position for the IPF-associated rare variant loci on chr20 (bp: 6232416662324601). Color for the top plot corresponds to SNP-DOC functional annotation and for the bottom plot, color corresponds to outlier by the Inner Tukey method and shape corresponds to outlier by the MAD method.
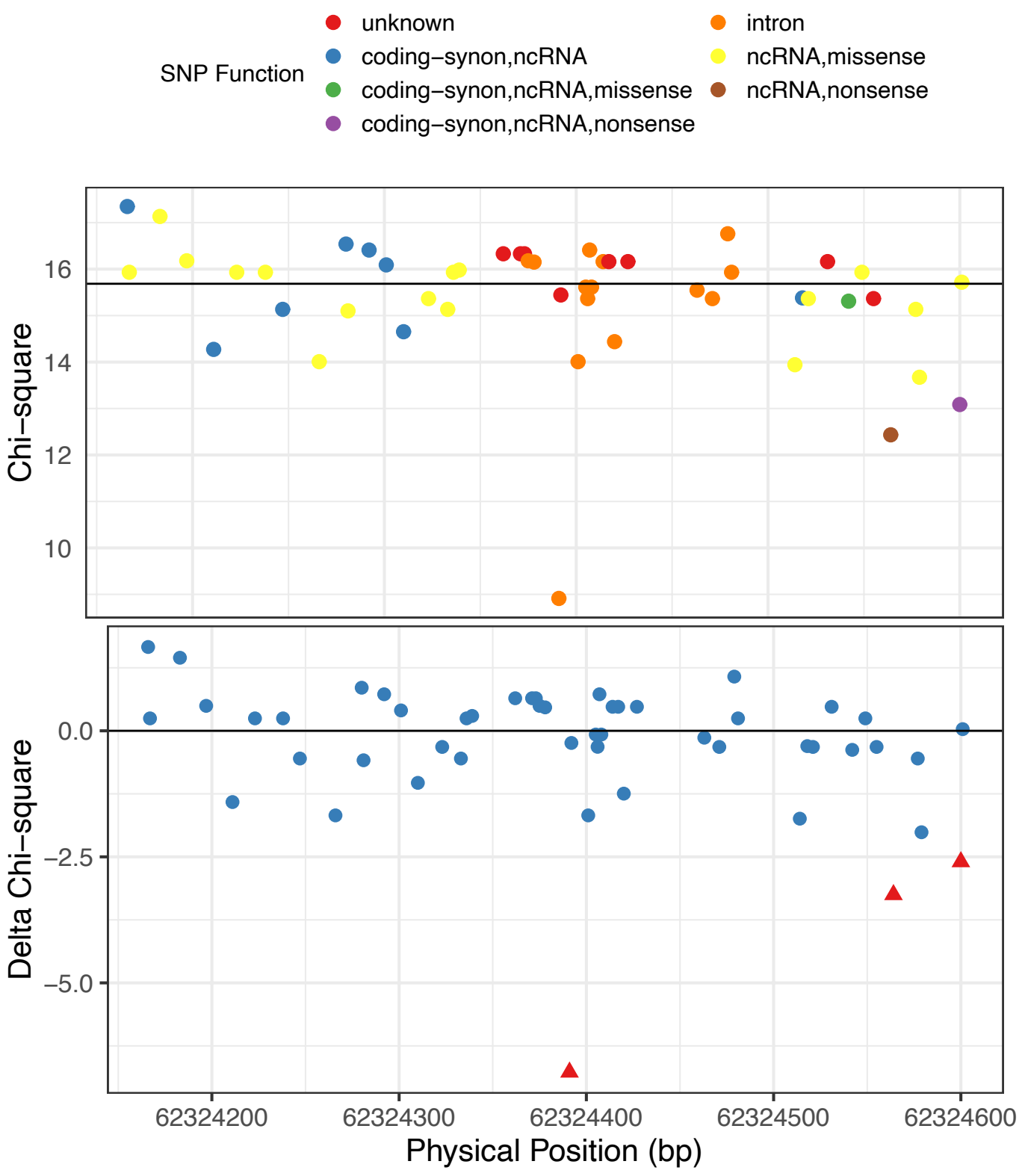

RIFT:Inner Tukey $\bullet$ Not Outlier $\bullet$ Outlier RIFT:MAD $\bullet$ Not Outlier $\Delta$ Outlier 